$$
\text { CONF-9711113-- }
$$

\title{
STRUCTURE OF NEUTRON-RICH NUCLEI
}

Witold Nazarewicz

Department of Physics, University of Tennessee, Knoxville, TN 37996

Physics Division, Oak Ridge National Laboratory*, Oak Ridge, TN 37831-6373

Institute of Theoretical Physics, Warsaw University, PL-00681, Warsaw, Poland

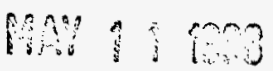

\author{
to be published in
Proceedings of International Conference on Fission
and Properties of Neutron-Rich Nuclei
Sanibel Island, Florida
November 13-15, 1997

की

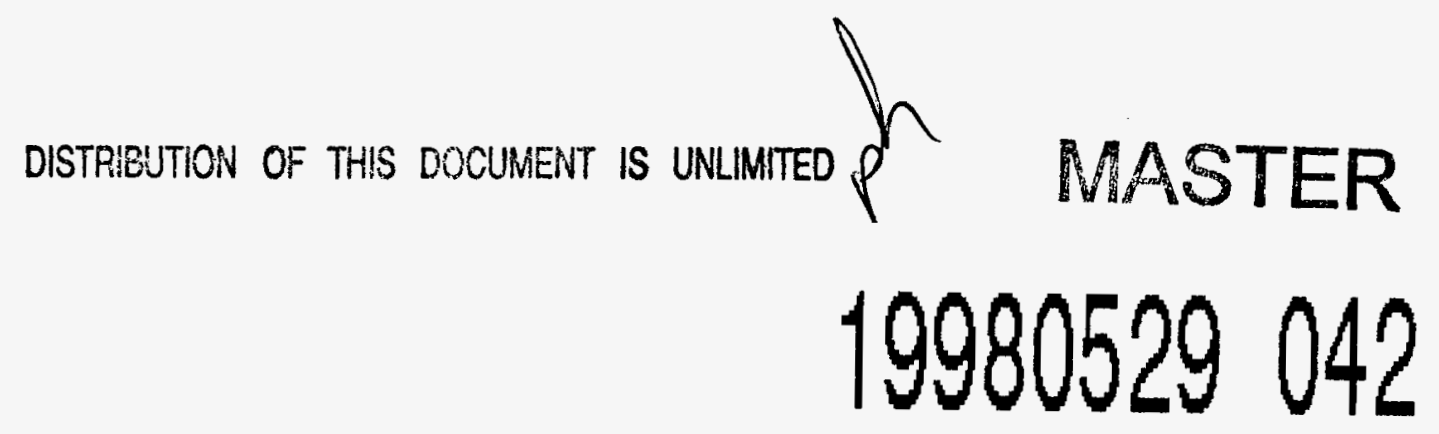

*Oak Ridge National Laboratory is managed by Lockheed Martin Energy Research Corp. for the U.S. Department of Energy under Contract No. DE-AC05-96OR22464.

"The submitted manuscript has been authored by a contractor of the U.S. Government under contract No. DE-AC05-96OR22464. Accordingly, the U.S. Government retains a nonexclusive, royalty-free license to publish or reproduce the published form of this contribution, or allow others to do so, for U.S. Government purposes." 


\section{DISCLAIMER}

This report was prepared as an account of work sponsored by an agency of the United States Government. Neither the United States Government nor any agency thereof, nor any of their employees, makes any warranty, express or implied, or assumes any legal liability or responsibility for the accuracy, completeness, or usefulness of any information, apparatus, product, or process disclosed, or represents that its use would not infringe privately owned rights. Reference herein to any specific commercial product, process, or service by trade name, trademark, manufacturer, or otherwise does not necessarily constitute or imply its endorsement, recommendation, or favoring by the United States Government or any agency thereof. The views and opinions of authors expressed herein do not necessarily state or reflect those of the United States Government or any agency thereof. 


\title{
STRUCTURE OF NEUTRON-RICH NUCLEI
}

\author{
WITOLD NAZAREWICZ \\ Department of Physics, University of Tennessee, Knoxville, TN 37996, USA \\ Physics Division, Oak Ridge National Laboratory, Oak Ridge, TN 37831, USA \\ Institute of Theoretical Physics, Warsaw University, PL-00681, Warsaw, Poland \\ E-mail: witek-nazarewicz@utk.edu
}

\begin{abstract}
One of the frontiers of today's nuclear science is the "journey to the limits": of atomic charge and nuclear mass, of neutron-to-proton ratio, and of angular momentum. The new data on exotic nuclei are expected to bring qualitatively new information about the fundamental properties of the nucleonic many-body system, the nature of the nuclear interaction, and nucleonic correlations at various energy-distance scales. In this talk, current developments in nuclear structure of neutron-rich nuclei are discussed from a theoretical perspective.
\end{abstract}

\section{Introduction}

The atomic nucleus is a fascinating many-body system bound by strong interaction. The building blocks of a nucleus (protons and neutrons) are themselves composite aggregates of quarks and gluons governed by quantum chromodynamics (QCD) - the fundamental theory of strong interaction. In the description of low-energy nuclear properties, one often simplifies the problem by replacing the complex subnucleonic structures by "effective" nucleons interacting through "effective" forces mediated by mesons. But even in this limit the dimension of the nuclear many-body problem is overwhelming. Nuclei are exceedingly difficult to describe; they contain too many nucleons to allow for an exact treatment and far too few to disregard finite-size effects. Also, the time scale characteristic of collective nuclear modes is close to the single-nucleonic time scale. This means that many concepts and methods applied successfully to other many-body systems, such as solids and molecules, cannot be adopted here.

\subsection{Nuclear forces}

The common theme for the whole field of nuclear structure is the problem of force: the one acting between two colliding nucleons, the one which produces exotic topologies in light nuclei, and that giving rise to the collective motion in heavy nuclei. The main challenges in understanding the nuclear force are shown in Fig. 1, in the context of the hadronic and nucleonic many-body problem. 


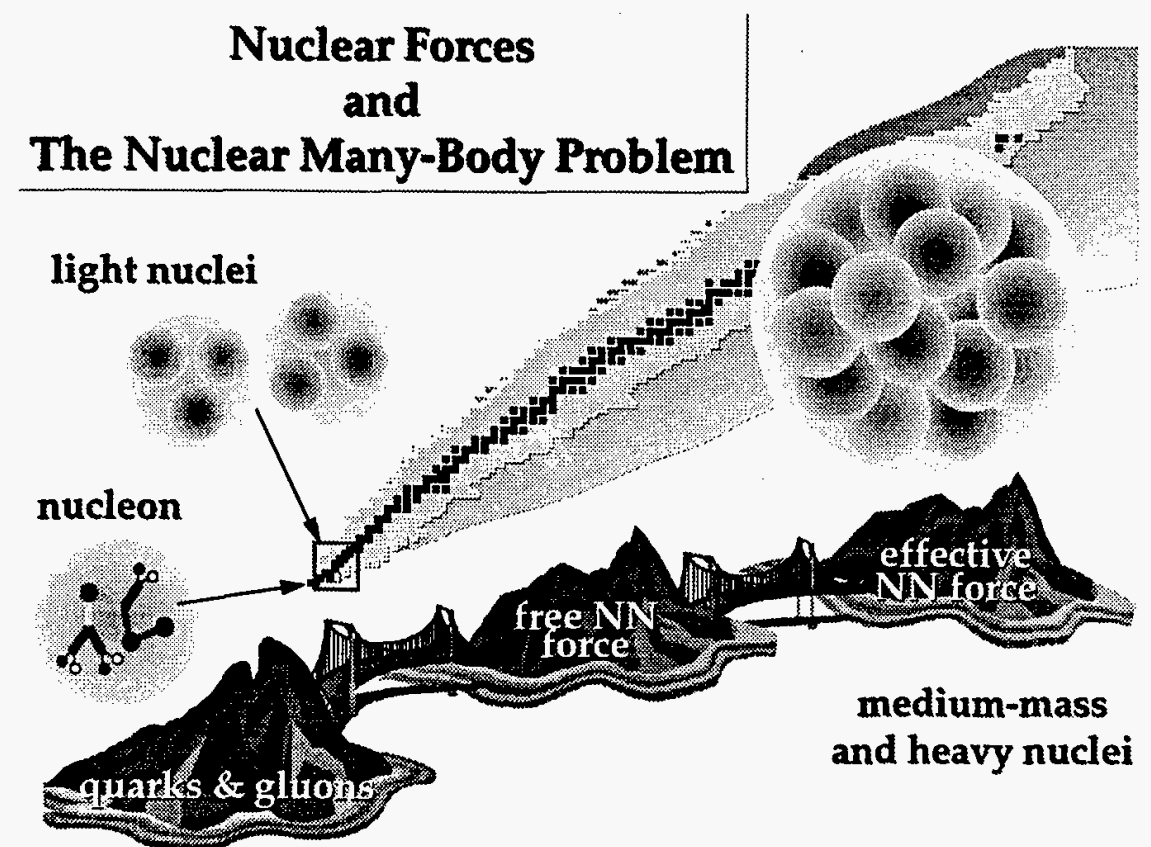

Figure 1: From hadrons to heavy nuclei: main challenges in understanding the nuclear force.

The low-energy interaction between nucleons has complicated spin-isospin dependence dictated by the hadron's substructure. One of the main challenges of nuclear science, indicated by the first bridge in Fig. 1, is the derivation of a nucleon-nucleon $(N N)$ interaction from the underlying quark-gluon dynamics of QCD. Experimentally, the $N N$ force can be studied by means of $N N$ scattering experiments. Examples of phenomenological parameterizations, based on the $N N$ scattering data, are the Bonn, Paris, Nijmegen, and Argonne potentials. While the long-range part of these free (but still effective!) $N N$ forces is well described by the one-pion exchange potential, their short-range behavior is not fully understood. Here, the quark-gluon degrees of freedom must be considered explicitly.

While the very light nuclei can nowadays be described as $A$-body clusters bound by a free $N N$ force (including higher-order interactions, such as a three-body force), the conceptual framework of larger nuclei is still that of the nuclear shell model. Here, the basic assumption is that the nucleons are moving almost independently in a mean potential obtained by averaging out the interactions between a single nucleon and all remaining $A-1$ protons and 
neutrons. The "effective" $N N$ interaction in the heavy nucleus used to determine the mean potential differs considerably from the free $N N$ force. In principle, it should be obtained by means of the complicated Brückner renormalization procedure which corrects the free $N N$ interaction for the effects due to the nuclear medium, and there has been some progress in this area. This challenging task is represented by the second bridge in Fig. 1. Many features of the effective interaction, such as short range; strong dependence on spins, isospins, and relative momenta of interacting nucleons; and the reduction of mass in the nuclear interior, have been extracted from experimental data.

Figure 1 illustrates the intellectual connection between the hadronic manybody problem (quark-gluon description of a nucleon) and the nucleonic manybody problem (nucleus as a system of $Z$ protons and $N$ neutrons). The free $N N$ force can be viewed as a residual interaction of the underlying quarkgluon dynamics of $Q C D$, similar to the intermolecular forces that stem from QED. Similarly, the effective $N N$ force in heavy nuclei can be derived from the effective free $N N$ interaction. It probably would be very naive to think of the behavior of a heavy nucleus directly in terms of the underlying quark-gluon dynamics, but, clearly, the understanding of the bridges in Fig. 1 will make this goal qualitatively possible.

\subsection{Recent developments in the nuclear many-body problem}

The best $N N$ force parameterizations not only describe the two-body on-shell properties but have been used in few-body and many-body calculations. Probably the most advanced few-body calculations today are the quantum Monte Carlo calculations with the Argonne-Urbana interaction for nuclei with $A \leq 7$ (Ref. ${ }^{1}$ ).

What about the shell-model treatment of heavier nuclei? In the past, shell-model calculations utilizing the concept of valence nucleons interacting in a restricted configuration space were limited to medium-mass nuclei owing to the rapid growth of the size of the model space. Today, this is still the case, although the conventional shell-model calculations employing realistic $N N$ interactions ${ }^{2,3}$ are becoming more and more efficient in handling large configuration spaces. The state-of-the-art shell-model studies of the $\dot{A}=47$ and $49 f p$ nuclei in the full $0 \hbar \omega$ space ${ }^{4}$ set the new standard in this area, although future progress is strongly limited by present-day computer resources. Actually, it took as long as two generations of hardware and software development to extend shell-model calculations from $A=44$ to $A=48$. (For the latter nucleus, the model spaces have dimensions of several millions.) The recently developed shell-model Monte Carlo method ${ }^{5}$ overcomes the dimension barrier; 
it represents the interaction between valence nucleons by auxiliary fields. Applications of the Monte Carlo approach to the nuclear shell model have been remarkably successful in describing many structural properties of heavy nuclei such as ${ }^{64} \mathrm{Ge}$ (Ref. ${ }^{6}$ ) or ${ }^{124} \mathrm{Xe}$ (Ref. ${ }^{7}$ ). (Here, the dimension of the model space is of the order of $10^{13}$ !)

For nuclei close to the magic ones, the low-energy properties depend primarily on the behavior of a few valence nucleons. However, for nuclei with many valence particles, the concept of valence nucleons is less useful, and the valence and inner-shell nucleons have to be treated on an equal footing. Many properties of these heavy systems are well described by means of self-consistent theories with the density-dependent effective $N N$ interaction. Here, the static mean-field description, based on the Hartree-Fock (HF), Hartree-Fock-Bogolyubov (HFB) method, or relativistic mean-field (RMF) theory, provides a useful starting point. Thanks to developments in computational techniques, the approaches employing microscopic effective interactions are now widely used and - in terms of their predictive power - favorably compare with results of more phenomenological macroscopic-microscopic models. Examples of recent large-scale, self-consistent, mean-field calculations include $\mathrm{HF}^{8}, \mathrm{HFB}^{9}$, and $\mathrm{RMF}^{10}$ studies of ground-state nuclear properties.

\subsection{Nuclear structure at the limits}

What are the frontiers of nuclear structure today? For light nuclei, one of such frontiers is physics at subfemtometer distances where the internal quark-gluon structures of nucleons overlap. For heavier nuclei, the frontiers are defined by the extremes of the neutron-to-proton ratio, atomic charge and nuclear mass, and angular momentum. Various aspects of physics at these limits are discussed in this volume. The tour to the limits is not only a quest for new and unexpected phenomena (sometimes dubbed as a "fishing expedition"), but they are expected as well to bring qualitatively new information about the effective $N N$ interaction and hence about the fundamental properties of the nucleonic many-body system. By exploring exotic nuclei, one can magnify certain terms of the Hamiltonian which are small in "normal" nuclei, thus difficult to test. The hope is that after probing these important interactions at the limits, we can later improve the description of normal nuclei (at ground states, close to the valley of beta stability, etc.).

In this talk, I would like to concentrate on the limit of large $N / Z$ ratios, i.e., physics of very large isospins. In addition to extending and improving our knowledge on known phenomena, nuclei with extreme neutron excess offer many prospects for entirely new physics. 


\section{Territory of Radioactive Nuclear Beams}

Physics of radioactive nuclear beams (RNB) is one of the main frontiers of nuclear science today. Experimentally, thanks to technological developments, we are on the verge of invading the territory of extreme $N / Z$ ratios in an unprecedented way ${ }^{11,12,13}$.

The nuclear landscape (the territory of the RNB physics) is shown in Fig. 2. Black squares indicate stable nuclei; there are less than 300 stable nuclei, or

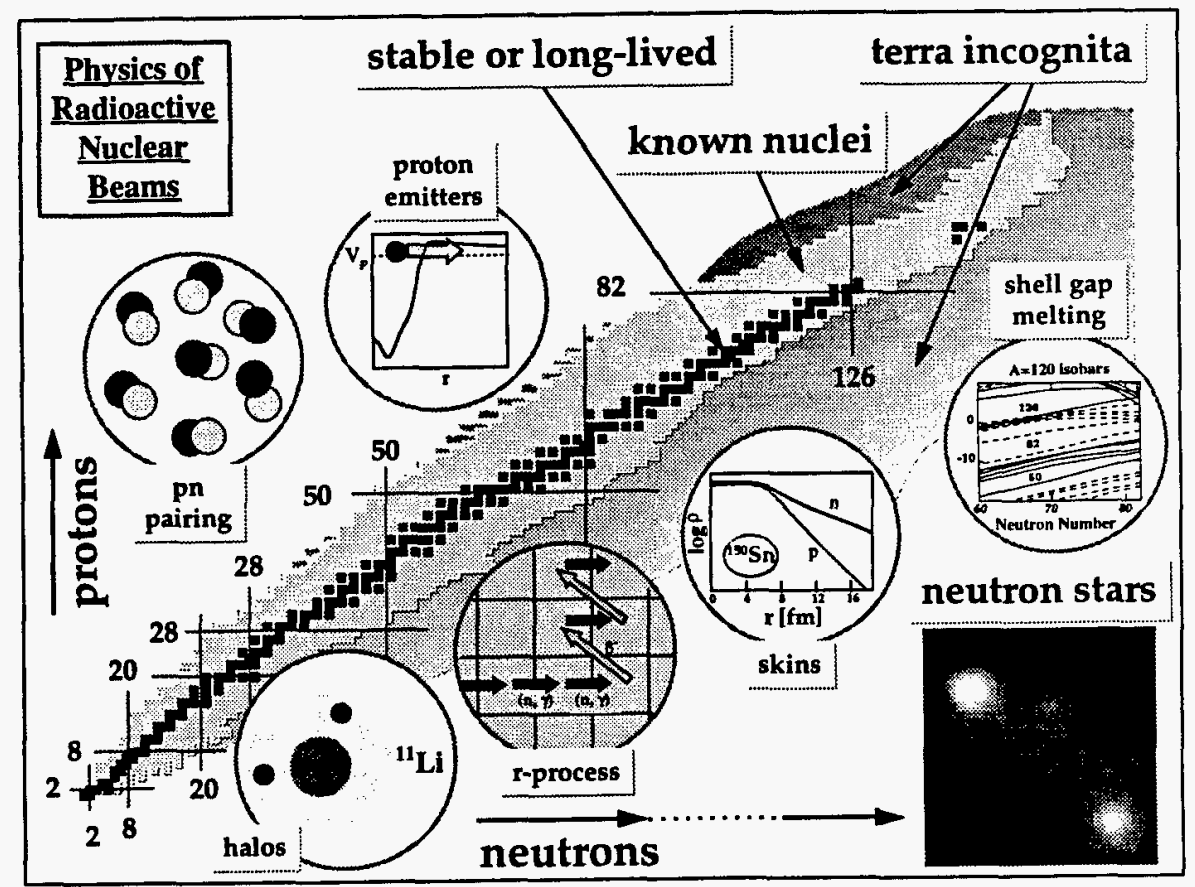

Figure 2: The nuclear landscape: territory of radioactive ion beam physics. Some of the important physics bullets are indicated schematically.

those long-lived, with half-lives comparable to or longer than the age of Earth. Some of the unstable nuclei can be found on Earth, some are man-made (actually, as many as $\sim 2,200$ nuclei have been produced in nuclear laboratories), and several thousand nuclei are the yet-unexplored exotic species. The yellow color indicates man-made nuclei that have been produced in laboratories and that live a shorter time. Moving away from the yellow area by adding either protons or neutrons, one finally reaches the particle drip lines where the 
nuclear binding ends. Many thousands of exotic radioactive nuclei with very small or very large $N / Z$ ratios are yet to be explored. In the $(Z, N)$ landscape, they form the "terra incognita" indicated in green. The nuclei beyond the drip lines are unbound to nucleon emission; that is, for those systems the strong interaction is unable to cluster $A$ nucleons as one nucleus. (Exceptions are neutron stars lying far far away from the neutron drip line. These giant nuclei, having masses of $\sim 1.4$ of the mass of the Sun and radii of $\sim 5$ miles, exist thanks to gravitation, an interaction which can safely be neglected in the description of "normal" nuclei.)

The uncharted regions of the $(N, Z)$ plane contain information that can answer many questions of fundamental importance for science: How many protons and neutrons can be clustered together by the strong interaction to form a bound nucleus? What are the properties of very short-lived exotic nuclei with an extreme neutron-to-proton ratio $N / Z$ ? What is the effective nucleonnucleon interaction in the nucleus having a very large neutron excess? There are also related questions in the field of nuclear astrophysics. Since radioactive nuclei are produced in many astrophysical sites, knowledge of their properties is crucial for our understanding of the underlying processes.

Where lie the particle drip lines? As seen in Fig. 2, the proton drip line is placed relatively close to the line of beta stability; hence it is easy to reach experimentally (actually, it has been delineated up to bismuth). However, since neutrons do not repel each other by the Coulomb force, many neutrons can be added to nuclei starting from the valley of stability. As a result, the "lever arm" separating the neutron drip line from the valley of stability is large and difficult to probe experimentally; except for the lightest nuclei, the bounds of the neutron stability are not known. The uncertainty in the limit of nuclear stability is illustrated by showing the unknown drip line as a dashed curve.

Nuclear life at extreme $N / Z$ ratios is different from that around the stability line. The unique structural factor is the weak binding; hence the closeness to the particle continuum.

\section{Theoretical Aspects of Physics at Extreme Isospins}

From a theoretical point of view, spectroscopy of exotic nuclei offers a unique test of those components of effective interactions that depend on the isospin degrees of freedom ${ }^{14}$. Effective interactions used to describe heavy nuclei are usually approximated by means of density-dependent forces with parameters that are usually fitted to stable nuclei and to selected properties of the infinite nuclear matter. Hence, it is by no means obvious that the isotopic trends far from stability, predicted by commonly used interactions (such as Skyrme or 
Gogny forces), are correct. In the models aiming at such an extrapolation, the important questions asked are: What is the $N / Z$ behavior of the two-body central force and the one-body spin-orbit force? Does the spin-orbit splitting strongly vary with $N / Z$ ? What is the importance of the effective mass (i.e., the non-locality of the force) for isotopic trends? What is the role of the medium effects (renormalization) and of the core polarization in the nuclear exterior (halo or skin region) where the nucleonic density is small?

Exotic nuclei are wonderful laboratories to study superconducting correlations. Here, the main questions pertaining to the problem of pairing force are: What is the microscopic origin of the pairing interaction? How can properties of the pairing force be tested? These questions are of considerable importance not only for nuclear physics but also for nuclear astrophysics and cosmology ${ }^{15}$. For instance, a better understanding of the density dependence of the nuclear pairing interaction is important for theories of superfluidity in neutron stars.

As mentioned above, the main theoretical challenge is the correct treatment of the particle continuum. For weakly bound nuclei, the Fermi energy lies very close to zero, and the decay channels must be taken into account explicitly. As a result, many cherished approaches of nuclear theory such as the conventional shell model, the pairing theory, or the macroscopic-microscopic approach must be modified. But there is also a splendid opportunity: the explicit coupling between bound states and continuum, and the presence of low-lying scattering states invite strong interplay and cross-fertilization between nuclear structure and reaction theory.

\section{Physics of Large Neutron Excess}

The intense current interest in experimental exploration of the neutron dripline region is driven not only by the substantial uncertainties in theoretical predictions of its location, but also by the expectation that qualitatively new features of nuclear structure will be discovered in this exotic territory. What makes neutron-rich nuclei so unusual? Firstly, they have very large sizes, as implied by their weak binding. Secondly, they are very diffused; their properties are greatly dominated by surface effects. Thirdly, they are very superconducting; the close-lying particle continuum provides a giant reservoir for scattered neutron Cooper pairs ${ }^{16,17}$. So, roughly speaking, they are large, fuzzy superconductors.

Just before the neutron drip line, neutrons occupy orbits outside the nuclear core that are spacially extended and, from Heisenberg's uncertainty principle, have low momentum. These states, called halos ${ }^{11}$, have radii that are up to several times that of the core (see Fig. 3). The halo region is a zone 


\section{Li: Borromean Halo Nucleus}
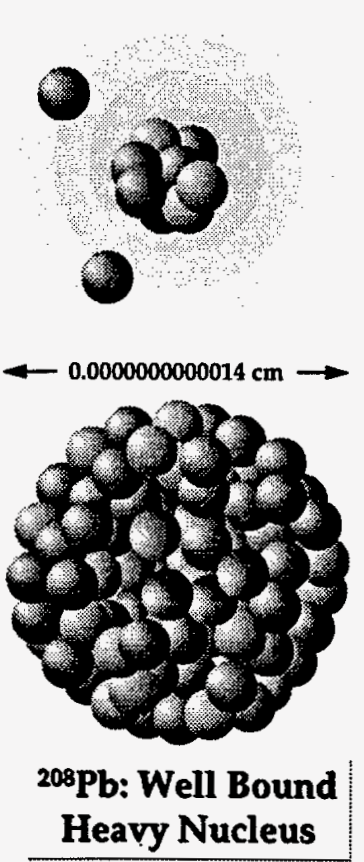
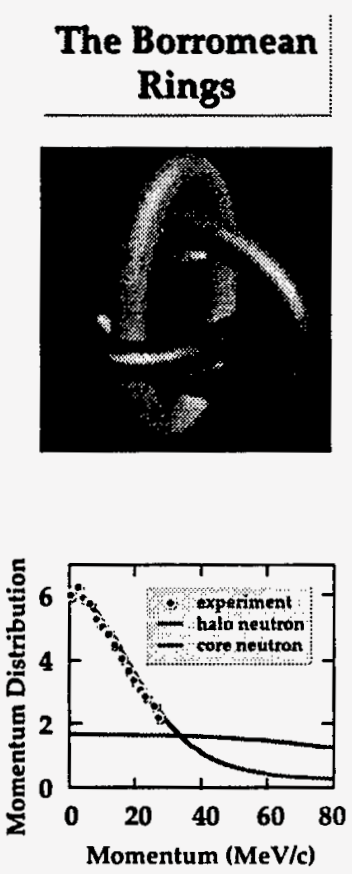

"Be: momentum distribution

Figure 3: Loosely bound halo nuclei such as ${ }^{11} \mathrm{Li}$ and ${ }^{11} \mathrm{Be}$ are unique few-body systems. A paradigm of the unexpected phenomena and unusual topologies that may occur in the vicinity of the neutron drip line is the nucleus ${ }^{11} \mathrm{Li}$ ( 3 protons and 8 neutrons), understood as a threebody halo consisting of two neutrons and a well-bound ${ }^{9} \mathrm{Li}$ core. Interestingly, while all three constituents of ${ }^{11} \mathrm{Li}$ form a bound system when placed together, the nuclear potential is not strong enough to bind any two of them separately. Hence the name borromean nuclei. (The name "borromean" comes from the Borromeo family of Renaissance Italy, who used three interlocking rings, with the property that if any one of them is removed, then all three separate, as their family coat of arms.) Due to very weak binding, the last neutrons in ${ }^{11} \mathrm{Li}$ occupy the same volume of space as the 208 nucleons in ${ }^{208} \mathrm{~Pb}$. The existence of halo structure in ${ }^{11} \mathrm{Be}$ has been deduced experimentally ${ }^{18}$ by studying the momentum distribution of the most weakly bound neutron. Compared to that for the well-bound neutron belonging to the ${ }^{10} \mathrm{Be}$ core, this distribution is exceptionally narrow, as expected from the Heisenberg uncertainty principle for a particle occupying an extended volume in space.

of weak binding in which quantum effects play a critical role in distributing 


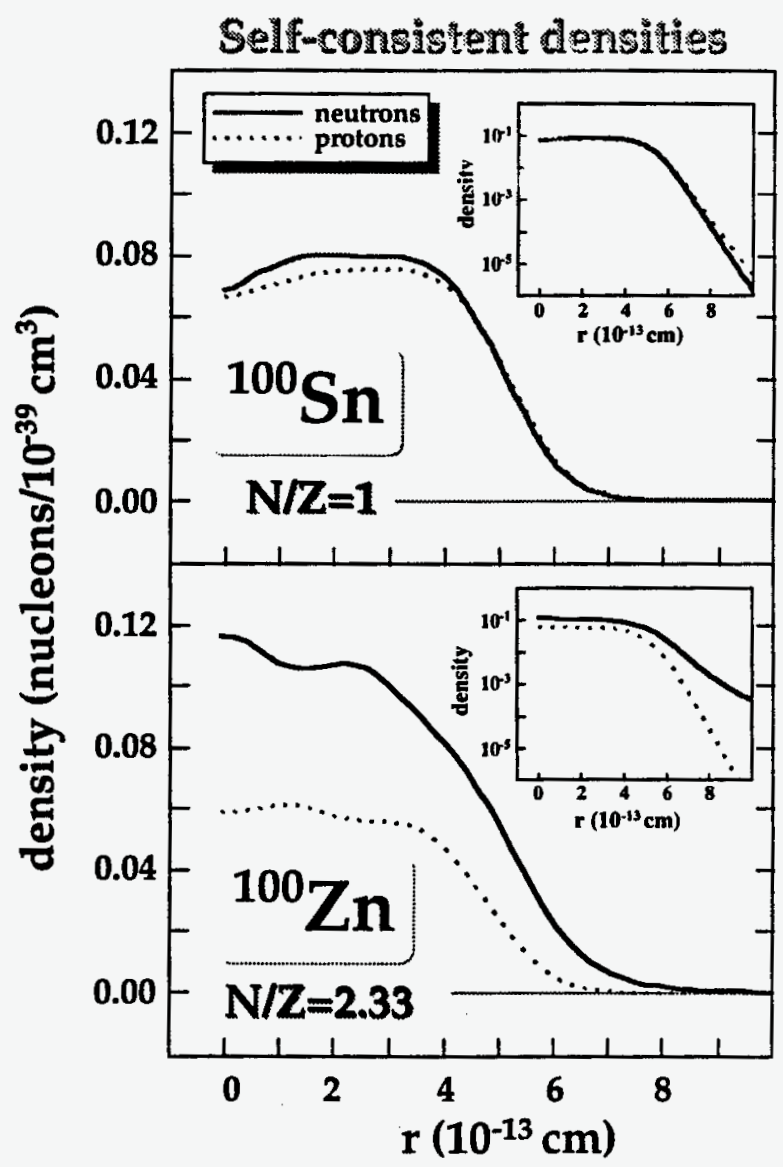

Figure 4: Proton and neutron density distributions in the $A=100$ nuclei calculated in the selfconsistent Hartree-Fock-Bogolyubov theory ${ }^{19}$. Top: ${ }^{100} \mathrm{Sn}$ (proton rich, $N / Z=1$ ); Bottom: ${ }^{100} \mathrm{Zn}$ (neutron rich, $N / Z=2.33$ ). Note how the neutrons extend much further out in ${ }^{100} \mathrm{Zn}$. This effect of skin is clearly seen in the logarithmic-scale plots in the insets. The small excess of neutrons in the interior of ${ }^{100} \mathrm{Sn}$ is compensated by the small excess of protons in the surface region. The diffused neutron density in ${ }^{100} \mathrm{Zn}$ gives rise to a very shallow shell-model potential.

nuclear density in regions not classically allowed. The heaviest neutron halo found so far ${ }^{20}$ is ${ }^{19} \mathrm{C}$, having 6 protons and 13 neutrons. Nuclei with two neutrons in their halo, such as ${ }^{11} \mathrm{Li}$, are unique objects because they break 
apart on removing one neutron. The attractive interaction between the two neutrons in the halo of these "Boromean" nuclei is essential for their binding. In the limit of extremely small binding energies of a pair of neutrons to a core nucleus, one will encounter giant halos with radii an order of magnitude larger than that of any stable nuclei ${ }^{21,22}$.

\section{Shapes and Excitation Modes of Halos \& Skins}

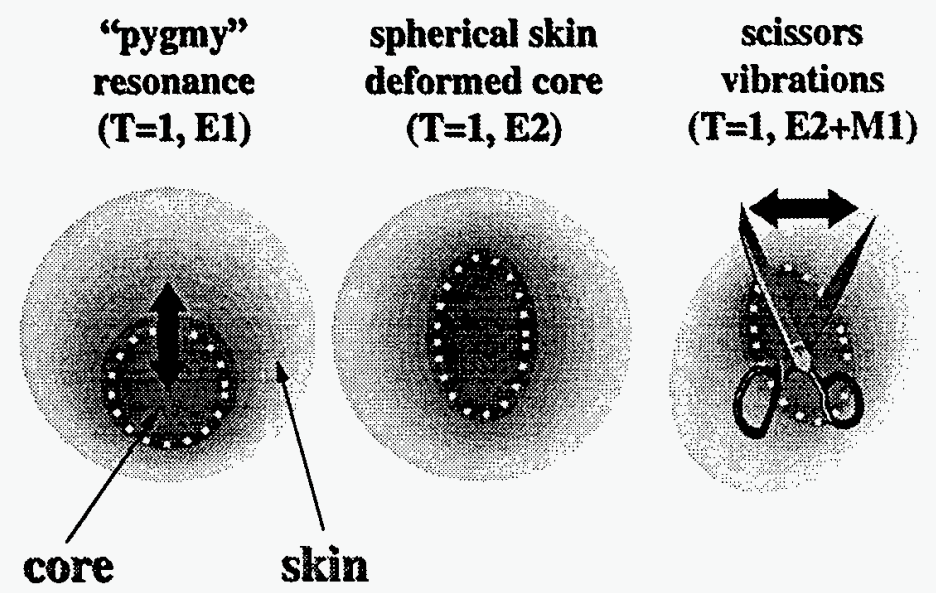

Figure 5: Schematic illustration of collective modes associated with the neutron skin. The left diagram shows the low-energy electric isovector dipole mode, dubbed "pygmy" resonance, associated with vibrations of the neutron skin (or halo) with respect to the core (indicated by a light dotted line). The middle diagram shows the system with very different quadrupole deformations of skin and core (here the core is deformed while the skin is spherical). The right diagram illustrates the angular vibrations of the deformed skin with respect to the deformed core (skin "scissors" mode).

In the heavier, neutron-rich nuclei, where the concept of mean field is better applicable, the separation into a "core" and "valence nucleons" seems less justified. However, also in these nuclei the weak neutron binding implies the existence of the neutron skin (i.e., a dramatic excess of neutrons at large distances). An example of neutron skin in ${ }^{100} \mathrm{Zn}$ is shown in Fig. 4.

In the skin region of heavier, very neutron-rich nuclei, one may find the opportunity to study in the laboratory nearly pure neutron matter at densities much less than the normal nuclear density. In addition, the existence of a neutron skin should lead to new collective vibration modes in such nuclei, in 
which, for example, the neutron skin may oscillate out of phase with a wellbound proton-neutron core. Some of these modes are schematically represented in Fig. 5.

\section{Nuclear Shell Structure}

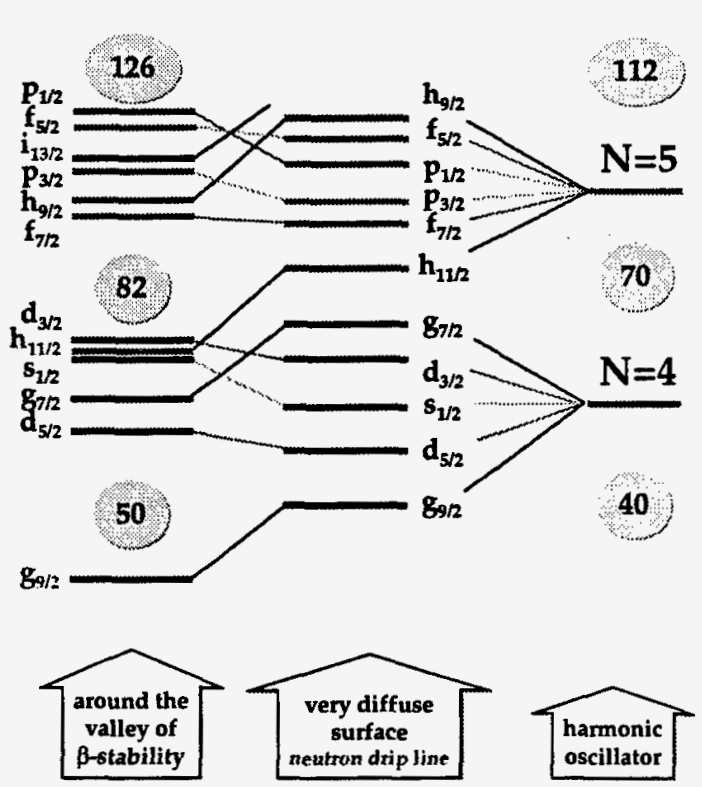

Figure 6: Left: Shell structure characteristic of nuclei close to the valley of stability. Middle: New shell structure characteristic of very shallow single-particle potentials in drip-line nuclei. It corresponds to a more uniform distribution of normal-parity orbits, and the unique-parity intruder orbit that reverts towards its parent shell. In the absence of a spin-orbit splitting (a significant reduction of spin-orbit coupling in neutron-rich nuclei has actually been predicted by some calculations), this single-particle spectrum is expected to approach the limit of the spherical harmonic oscillator (shown in the right panel). (From Ref. ${ }^{17}$.)

\subsection{Nuclear shell structure far from stability}

The structure of nuclei is expected to change significantly as the limit of nuclear stability is approached in neutron excess. Due to the systematic variation in 
the spatial distribution of nucleonic densities and the increased importance of the pairing field, the average nucleonic potential is modified ${ }^{19}$. This results in a new shell structure characterized by a more uniform distribution of normalparity orbits and the unique-parity intruder orbit which reverts towards its parent shell (see Fig. 6).

The quenching of shell effects manifests itself in the behavior of twoneutron separation energies $S_{2 n}$. This is illustrated in Fig. 7, which displays the two-neutron separation energies for the $N=80,82,84$, and 86 spherical even-even isotones calculated in the HFB model with the SkP and SLy 4 effective interactions. The large $N=82$ magic gap, clearly seen in the nuclei close to the stability valley and to the proton drip line, gradually closes down when approaching the neutron drip line. As discussed in, e.g., Refs. ${ }^{23,24}$, this has

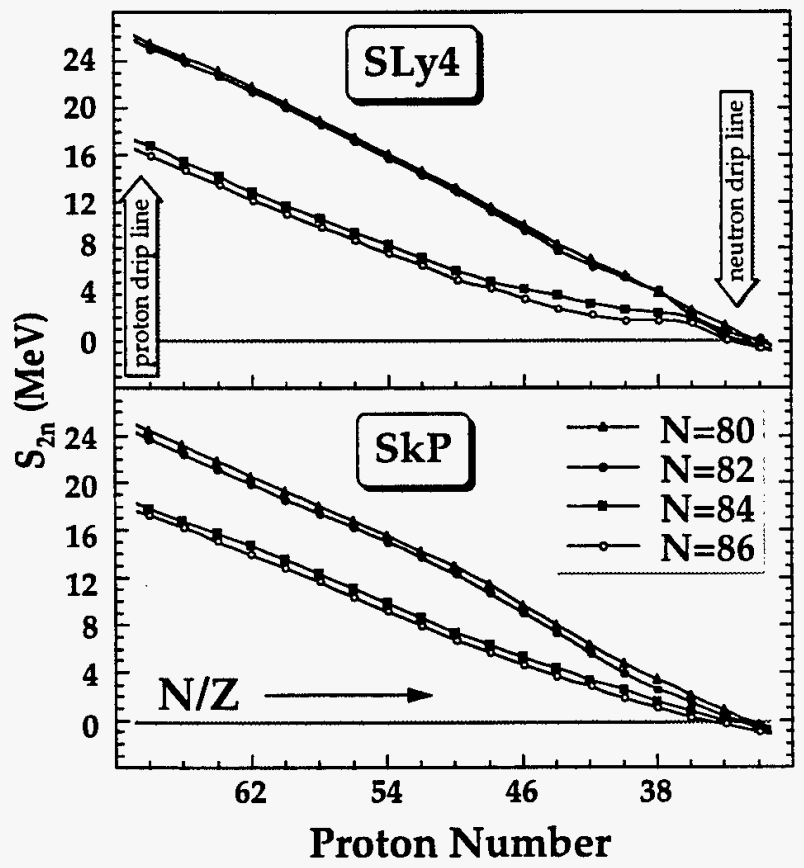

Figure 7: Two-neutron separation energies for the $N=80,82,84$, and 86 spherical even-even isotones calculated in the $\mathrm{HFB}+\mathrm{SkP}$ and $\mathrm{HFB}+\mathrm{SLy} 4^{\delta}$ models as functions of the proton number. The arrows indicate the proximity of neutron and proton drip lines for small and large proton numbers, respectively. (From Ref. ${ }^{14}$.)

important consequences for the $r$-process and the stellar nucleosynthesis. 


\section{Major World Wide Radioactive Beam Facilities}

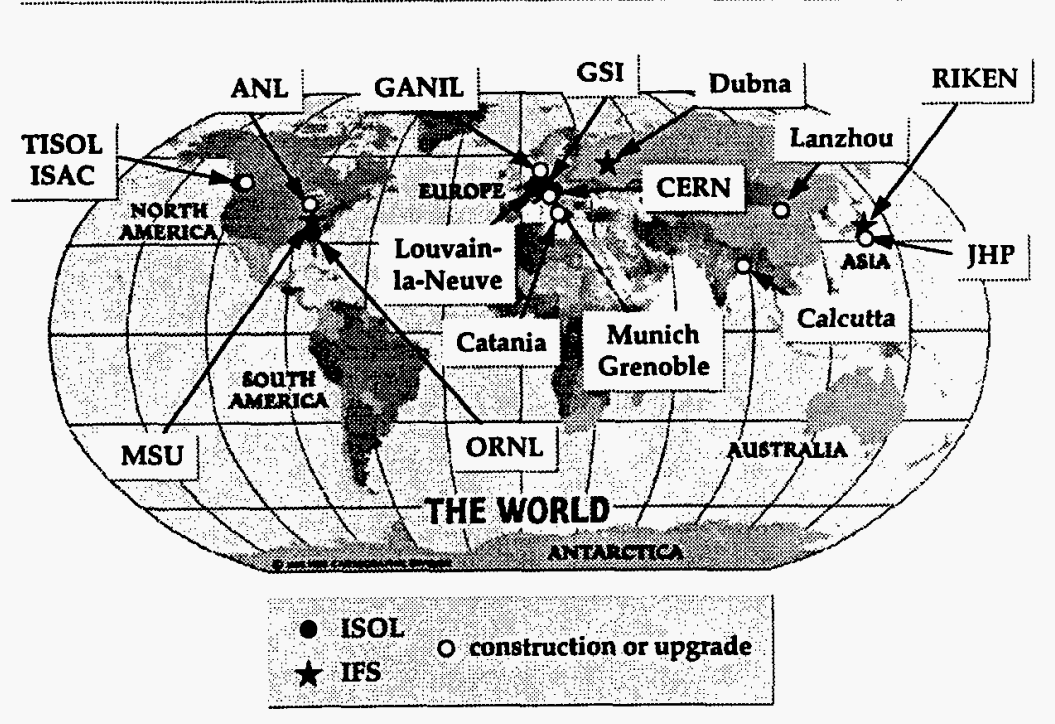

Figure 8: Worldwide efforts in radioactive nuclear beams.

\subsection{Radioactive nuclear beams}

Many of the current limitations in nuclear physics studies promise to be lifted by the developing field of radioactive nuclear ion beam experimentation. Advances in accelerator, ion source, and mass separator technology have, over the past 20 years, greatly improved our ability to produce, separate, and accelerate radioactive ions. One of the indications of the potential of this field is the large international interest in the development of facilities with RNB capabilities. At present there are only a few laboratories with radioactive ion beam capabilities. However, the prospects for new experiments and the success of the current programs have lead to a number of RNB facilities under development and a number of further proposals. Figure 8 illustrates the worldwide effort in radioactive nuclear beams. Facilities which are now operational are labeled with a star (for In-flight separation, IFS) or full circle (for Isotope separation on line, ISOL), while facilities under construction are illustrated with an open circle. Other facilities which have been proposed, or are under discussion, are indicated by a small closed circle. 
In trying to see the phenomena of a "new physics", we should ask the fundamental question of "how far is far"? Experiments with radioactive beams are going to be long and difficult, and many examples of nuclear exotica discussed in this paper (especially those concerning very neutron-rich systems) are clearly out of reach, even assuming most optimistic experimental scenarios ${ }^{13}$. The hope is, however, that some of the effects associated with the loose binding will be seen as deviations from smooth systematic trends or will show up at higher excitation energies closer to the particle threshold, as in the example of the analog states ${ }^{25,26}$. Theoretically, we are bound to adopt the strategy of going to the extreme values of $N / Z$ in order to identify the qualitatively new phenomena, and then back down to experimentally achievable regions to see whether these phenomena can actually be observed. There is very little doubt that we are on the verge of the most fascinating fishing expedition.

\section{Acknowledgments}

This research was supported by the U.S. Department of Energy under Contract No. DE-FG02-96ER40963 with University of Tennessee. Oak Ridge National Laboratory is managed for the U.S. Department of Energy by Lockheed Martin Energy Research Corporation under Contract No. DE-AC05-96OR22464.

1. B.S. Pudliner, V.R. Pandharipande, J. Carlson, S.C. Pieper, and R.B. Wiringa, Phys. Rev. C56, 1720 (1997).

2. F. Andreozzi, L. Coraggio, A. Covello, A. Gargano, T.T.S. Kuo, Z.B. Li, and A. Porrino, Phys. Rev. C54, 1636 (1996).

3. P. Navratil, M.Thoresen, and B.R. Barrett, Phys. Rev. C55, R573 (1997).

4. G. Martinez-Pinedo, A.P. Zuker, A. Poves, and E. Caurier, Phys. Rev. C55, 187 (1997).

5. S.E. Koonin, D.J. Dean, and K. Langanke, Phys. Rep. 278, 1 (1997).

6. M. Honma, T. Mizusaki, and T. Otsuka, Phys. Rev. Lett. 77, 3315 (1996).

7. Y. Alhassid, G.F. Bertsch, D.J. Dean, and S.E.Koonin, Phys. Rev. Lett. 77, 1444 (1996).

8. N. Tajima, S. Takahara, and N. Onishi, Nucl. Phys. A603, 23 (1996).

9. J. Dobaczewski, W. Nazarewicz, and T.R. Werner, Z. Phys. A354, 27 (1996).

10. D. Hirata, K. Sumiyoshi, I. Tanihata, Y. Sugahara, T. Tachibana, and H. Toki, Nucl. Phys. A616, 438c (1997).

11. P.G. Hansen, A.S. Jensen, and B. Jonson, Annu. Rev. Nucl. Part. Phys. 45, 591 (1995). 
12. W. Nazarewicz, B. Sherril, I. Tanihata, and P. Van Duppen, Nuclear Physics News 6, 17 (1996).

13. Scientific Opportunities With an Advanced ISOL Facility, Report, November 1997.

14. J. Dobaczewski and W. Nazarewicz, Phil. Transactions, in press, 1997; nucl-th/9707049.

15. C.J. Pethick and D.G. Ravenhall, Annu. Rev. Nucl. Part. Sci. 45, 429 (1995).

16. J. Dobaczewski, H. Flocard, and J. Treiner, Nucl. Phys. A422, 103 (1984).

17. J. Dobaczewski, W. Nazarewicz, T.R. Werner, J.-F. Berger, C.R. Chinn, and J. Dechargé, Phys. Rev. C53, 2809 (1996).

18. J.H. Kelley, S.M. Austin, R.A. Kryger, D.J. Morrissey, N.A. Orr, B.M. Sherrill, M. Thoennessen, J.S. Winfield, J.A. Winger, and B.M. Young, Phys. Rev. Lett. 74, 30 (1995).

19. J. Dobaczewski, I. Hamamoto, W. Nazarewicz, and J.A. Sheikh, Phys. Rev. Lett. 72, 981 (1994).

20. D. Bazin, B.A. Brown, J. Brown, M. Fauerbach, M. Hellstrom, S.E. Hirzebruch, J.H. Kelley, R.A. Kryger, D.J. Morrissey, R. Pfaff, C.F. Powell, B.M. Sherrill, and M. Thoennessen, Phys. Rev. Lett. 74, 3569 (1995).

21. V. Efimov, Phys. Lett. 33B, 563 (1970).

22. D.V. Fedorov, A.S. Jensen, and K. Riisager, Phys. Rev. Lett. 73, 2817 (1994).

23. B. Chen, J. Dobaczewski, K.-L. Kratz, K. Langanke, B. Pfeiffer, F.-K. Thielemann, and P. Vogel, Phys. Lett. B355, 37 (1995).

24. B. Pfeiffer, K.-L. Kratz, and F.-K. Thielemann, Z. Phys. A357, 235 (1997).

25. R.G. Thomas, Phys. Rev. 81, 148 (1951); 88, 1109 (1952).

26. J.B. Ehrman, Phys. Rev. 81, 412 (1951). 


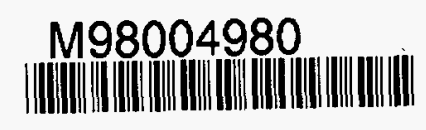

Report Number (14) ORNL/CP $-97 / 60$
CONF-9711113

Publ. Date (11) $1997 / 1$

Sponsor Code (18) DOE/ER , XF

UC Category (19) UC-400, DOE/ER 